\title{
Cardiac Injury Biomarkers and the Risk of Death in Patients with COVID-19: A Systematic Review and Meta-Analysis
}

\author{
Sami H. Alzahrani $\mathbb{i D}^{1}$ and Mohammed W. Al-Rabia $\mathbb{i D}^{2}$ \\ ${ }^{1}$ Family Medicine Department, Faculty of Medicine, King Abdulaziz University, Jeddah, Saudi Arabia \\ ${ }^{2}$ Department of Medical Microbiology and Parasitology, Faculty of Medicine, King Abdulaziz University, Jeddah, Saudi Arabia \\ Correspondence should be addressed to Sami H. Alzahrani; drsamihz@gmail.com
}

Received 4 July 2020; Revised 18 February 2021; Accepted 4 March 2021; Published 18 March 2021

Academic Editor: Giovanni Battista Forleo

Copyright (c) 2021 Sami H. Alzahrani and Mohammed W. Al-Rabia. This is an open access article distributed under the Creative Commons Attribution License, which permits unrestricted use, distribution, and reproduction in any medium, provided the original work is properly cited.

\begin{abstract}
Background. Cardiac complications may develop in a proportion of patients with the novel coronavirus disease (COVID-19), which may influence their prognosis. Objectives. To assess the role of cardiac injury biomarkers measured on admission and during hospitalization as risk factors for subsequent death in COVID-19 patients. Methods. A systematic review and meta-analysis was carried out involving cohort studies that compared the levels of cardiac injury biomarkers in surviving and dead COVID-19 patients. Cardiac injury is defined as an elevation of the definitive markers (cardiac troponin (cTnI and cTnT) and N-terminal proB-type natriuretic peptide (NT-proBNP)) above the 99th percentile upper reference limit. Secondary markers included creatine kinase-myocardial bound (CK-MB), myoglobin, interleukin-6 (IL-6), and C-reactive protein (CRP). The risk of death and the differences in marker concentrations were analyzed using risk ratios (RRs) and standardized mean differences (SMDs), respectively. Results. Nine studies met the inclusion criteria (1799 patients, $53.36 \%$ males, $20.62 \%$ with cardiac injury). The risk of death was significantly higher in patients with elevated cTn than those with normal biomarker levels $(\mathrm{RR}=5.28, P<0.0001)$. Compared to survivors, dead patients had higher levels of cTn ( $\mathrm{SMD}=2.15, P=0.001)$, IL-6 $(\mathrm{SMD}=3.13, P=0.03)$, hs-CRP $(\mathrm{SMD}=2.78, P<0.0001)$, and $\mathrm{CK}-\mathrm{MB}(\mathrm{SMD}=0.97, P<0.0001)$ on admission and a significant rise of plasma cTnT during hospitalization. Conclusion. COVID-19 patients with elevated cTn on admission, possibly due to immune-mediated myocardial injury, are at increased risk for mortality. This requires further radiographic investigations, close monitoring, and aggressive care to reduce the risk of severe complications and death.
\end{abstract}

\section{Introduction}

The world has experienced an unprecedented pandemic of the novel severe acute respiratory syndrome coronavirus-2 (SARS-CoV-2) since the last days of 2019 when unexplained cases of lower respiratory tract infections have been reported in the Huanan Seafood Market, Wuhan, China [1]. The causative virus belongs to the Coronaviridae family, which comprises of a large group of coronaviruses (CoVs) that affect animals and humans. Similar to the highly identical virus SARS-CoV [2], the envelope spike protein (S protein) of SARS-CoV-2 binds to the angiotensin-converting enzyme 2 (ACE-2) receptors [3]. However, the novel virus has a 10to 20 -fold higher affinity for binding to its specific receptors than SARS-CoV [4], which may partly explain the widespread pattern among different populations. SARS-CoV-2 is transmitted from human to human, primarily via droplets and close contact [5]. The clinical spectrum of patient presentation ranges from the absence of apparent symptoms to severe respiratory failure [6]. Symptomatic patients usually present with fever, dry cough, dyspnea, myalgia, and fatigue; additionally, the majority of patients experience pneumonia.

In addition to the dominant respiratory symptoms, some COVID-19 patients might have severe cardiovascular damage. A clinical bulletin released by the American College of Cardiology [7] indicated the relevance of cardiac complications of the novel virus based on the fact that $12 \%$ of 
COVID-19 patients had had coassociated cardiac injury [6]. The risk of death might also be influenced by the history of a cardiovascular disease, and a proportion of patients may die as a result of cardiovascular deterioration along the course of the disease. Therefore, the role of cardiovascular biomarkers as predictors of future cardiac events might be significant in risk stratification on admission and during hospitalization. In particular, two isoforms of cardiac troponin (cTn), including cTnI and cTnT, have been widely used for the diagnosis of acute coronary syndrome [8]. In addition, natriuretic peptides, especially the $\mathrm{N}$-terminal pro-B-type natriuretic peptide (NT-proBNP), are important biomarkers for diagnosis and prognosis of heart failure [9]. These laboratory markers have been reported in recent studies demonstrating the clinical characteristics of COVID-19 patients; however, few reports have emphasized the relevance of cardiac injury with the risk of death. The present systematic review and meta-analysis aimed to assess the association between the abnormal levels of cardiac injury biomarkers measured on admission and the subsequent risk of death among COVID-19 patients. Besides, we sought to explore the dynamic changes that have occurred during hospitalization in survived and deceased patients.

\section{Methods}

A systematic review and meta-analysis was conducted following the recommended guidelines of the Preferred Reporting Items for Systematic Reviews and Meta-Analyses (PRISMA) [10]. Eligible articles included retrospective and prospective cohort studies which reported the baseline laboratory findings of cardiac injury biomarkers in survived and deceased patients who had been diagnosed with COVID-19 according to the interim guidelines of the WHO [11] and had been confirmed using the reverse transcriptase polymerase chain reaction (RT-PCR) assay. Peer-reviewed and preprint articles were included. Definitive myocardial injury biomarkers included cTnI, cTnT, and NT-proBNP [12], and their elevated values in the serum (above the 99th percentile upper reference limit) have indicated cardiac injury. In addition to baseline values, dynamic laboratory findings of the definitive biomarkers during hospitalization in survived and nonsurvived groups were investigated. Moreover, baseline creatine kinase-myocardial bound (CK$\mathrm{MB})$, myoglobin, and the inflammatory parameters interleukin-6 (IL-6) and C-reactive protein (CRP) were considered as secondary biomarkers [12]. Studies recruiting specific populations of patients, such as critically ill and elderly, were excluded to optimize the generalizability of the results and to exclude the effects of aberrant cardiovascular biomarkers in such populations on the primary outcomes (mortality or survival). Review articles, case reports, and articles written in non-English language were ineligible.

Two independent authors, Dr. S.A. and M.A., performed the main search process in PubMed, Embase, and Google Scholar as of April 11, 2020. The date of publication was limited to 2020 when the outbreak emerged. Specific keywords were used in combination with Boolean operators (such as OR and AND); the strategy used in the PubMed database is provided in Appendix. The obtained records were exported to a specific program (Endnote X9, build $12,062)$ to organize references and delete duplicate records. The titles and abstracts of all the records were screened thoroughly, and the full-text versions of eligible studies were downloaded according to the availability of primary outcomes. Data extraction was performed in a spreadsheet (Microsoft Excel 2016) designed specifically for the study. Any disagreement between data collectors regarding study inclusion and data collection was resolved by discussion. The extracted data included (1) characteristics of the studies: the last name of the first author, country, setting, design, and period of data collection; (2) participants' data: the number of patients, gender, mean age of the total cohort, comorbidities at presentation, and the frequency of nonsurvival; (3) primary cardiac markers: baseline and in-hospital quantitative values of cTnI, cTnT, or NT-proBNP (pg/mL) and/or the frequency of patients with elevated biomarkers in serum; and (4) secondary biomarkers: baseline high-sensitivity CRP (hs-CRP) (mg/L), CK-MB (U/L), IL-6 (pg/mL), and myoglobin $(\mathrm{ng} / \mathrm{mL})$.

Numerical variables expressed as medians and interquartile ranges were converted to means and standard deviations (SDs) as indicated by Wan et al. [13]. The graphically represented data were converted to their respective numerical values after setting the accurate coordinates on images using GetData Graph Digitizer 2.26. The pooled case fatality rate and the prevalence of cardiac injury, as well as their respective $95 \%$ confidence intervals (95\% CIs), were computed based on the weight of each study and the total number of patients. The standard error (SE) was calculated using the following formula: $\mathrm{SE}=\sqrt{p(1-p) / n}$, where $p$ indicates the prevalence and $n$ indicates the sample size. All the quantitative and qualitative data were entered and analyzed in Review Manager software (version 5.3). For the analyses of the frequency of patients with elevated or normal cTn values (dichotomous variables), odds ratio and 95\% CI expressed the difference in baseline comorbid conditions, whereas risk ratio (RR) and 95\% CI were applied to assess the risk of death between both groups. Standardized mean differences (SMDs) and 95\% CIs were used to analyze the difference in the quantitative values of cardiac biomarkers between survivors and nonsurvivors. The Mantel-Haenszel formula and inverse variance calculations were applied to the qualitative and quantitative analyses, respectively. The pooled effect estimates were computed using random-effects models if the heterogeneity between studies was significant $\left(I^{2}>50 \%\right)$; otherwise, a fixed-effects model was applied. Funnel plots were generated to explore publication bias.

The methodological quality was appraised by two authors (S.A. and M.A.) using the Strengthening the Reporting of Observational Studies in Epidemiology (STROBE) criteria for cohort studies [14], in which a score between 0 and 22 is given to each individual study based on a checklist of specific items related to the assessment of the title/abstract, introduction, methods, results, discussion, and other information. A STROBE score of $<12$ or $12-17$ indicated a poor or moderate methodological quality, respectively. 


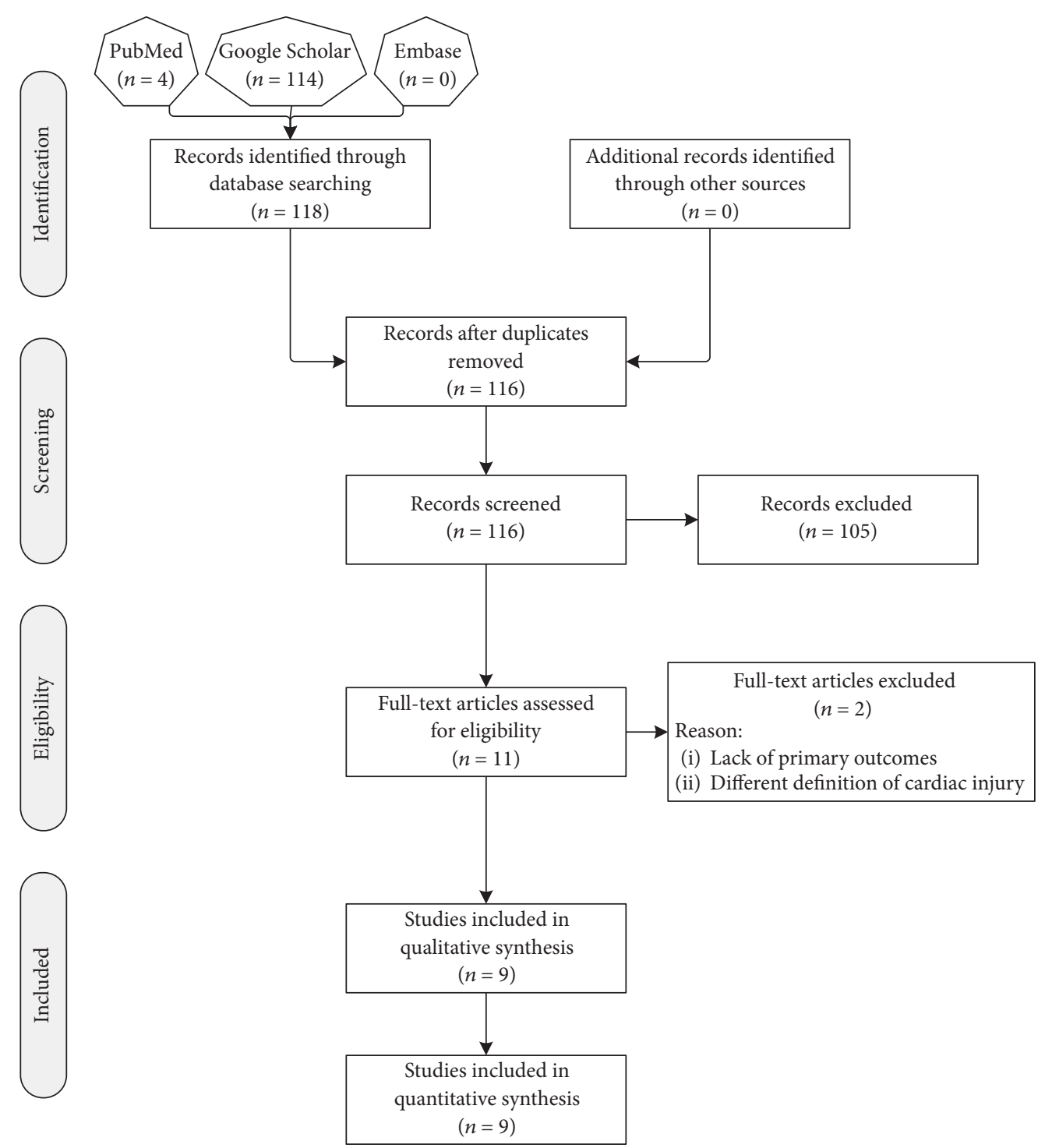

FIGURE 1: Flow chart showing the search process employed in the current study.

\section{Results}

3.1. Results of the Search Process. Figure 1 shows the results of the search strategy across different databases. Initially, 118 records were identified, of which two duplicates were omitted. The results of the screening process revealed a total of 11 studies meeting the eligibility criteria. However, after checking the full versions of eligible articles, two studies were excluded due to lack of primary outcomes in surviving and nonsurviving groups [15] and defining cardiac injury as elevated serum cardiac biomarkers above the 66th percentile upper reference limit [16]. Therefore, nine studies were formally included in the qualitative and quantitative analyses [17-25].

3.2. Characteristics of the Included Studies. The characteristics of the included studies are provided in Table 1. All studies were carried out in China, and they included retrospective analyses of medical records to collect patient data.
Data collection was performed over a period between 21 and 52 days. A total of 1799 patients were included $(53.36 \%$ males), and sample sizes ranged between 48 and 416 patients in a study. A history of hypertension was reported in $31.85 \%$ of patients, diabetes in $15.45 \%$, coronary heart disease (CHD) in $9.41 \%$, chronic obstructive pulmonary disease (COPD) in $3.88 \%$, and malignancy in $3.41 \%$. The methodological qualities of four studies $[19,21,23,25]$ were judged as "moderate," whereas the remainder were of high methodological quality (Table 1). The overall weighted case fatality rate among hospitalized patients was $24.01 \%$ (95\% CI 17.70-30.32, $I^{2}=91 \%$, Supplementary Figure S1).

3.3. Levels of Cardiac Injury Biomarkers in Surviving and Deceased Patients. Data regarding the mean cTn levels in survivors and nonsurvivors were available in six studies (1017 patients) [17, 19-21, 23, 24]. These included highsensitivity cTnI (hs-cTnI) and cTnT. On admission, patients who did not survive had had significantly higher 
TABle 1: Characteristics of the included studies.

\begin{tabular}{|c|c|c|c|c|c|c|c|c|c|c|}
\hline Author & $\begin{array}{l}\text { Design } \\
\text { (country) }\end{array}$ & Settings & $\begin{array}{c}\text { Period of data } \\
\text { collection } \\
\text { (days) }\end{array}$ & $\begin{array}{l}\text { Sample } \\
\text { size }\end{array}$ & $\begin{array}{r}\mathrm{Ge} \\
\text { Male }\end{array}$ & $\begin{array}{l}\text { ender } \\
\text { Female }\end{array}$ & Age & Survived & Deaths & $\begin{array}{c}\text { STROBE } \\
\text { score }\end{array}$ \\
\hline $\begin{array}{l}\text { Chen } \\
\text { et al. [17] }\end{array}$ & $\begin{array}{c}\mathrm{RC} \\
\text { (China) }\end{array}$ & $\begin{array}{c}\text { Zhongnan Hospital and } \\
\text { the Seventh Hospital of } \\
\text { Wuhan city }\end{array}$ & 45 & 123 & 61 & 62 & $57.79 \pm 15.32$ & 92 & 31 & 19 \\
\hline $\begin{array}{l}\text { Du et al. } \\
{[25]}\end{array}$ & $\begin{array}{c}\mathrm{RC} \\
\text { (China) }\end{array}$ & $\begin{array}{c}\text { Wuhan Pulmonary } \\
\text { Hospital }\end{array}$ & 43 & 179 & 97 & 82 & $57.60 \pm 13.70$ & 158 & 21 & 16 \\
\hline $\begin{array}{l}\text { Guo et al. } \\
{[18]}\end{array}$ & $\begin{array}{c}\mathrm{RC} \\
\text { (China) }\end{array}$ & $\begin{array}{l}\text { The Seventh Hospital, } \\
\text { Wuhan }\end{array}$ & 31 & 187 & 91 & 96 & $58.50 \pm 14.66$ & 144 & 43 & 20 \\
\hline $\begin{array}{l}\text { Li et al. } \\
{[19]}\end{array}$ & $\begin{array}{c}\mathrm{RC} \\
\text { (China) }\end{array}$ & Tongji Hospital, Wuhan & 35 & 102 & 59 & 43 & $57.25 \pm 4.99$ & 87 & 15 & 15 \\
\hline Luo et al. & $\begin{array}{c}\mathrm{RC} \\
\text { (China) }\end{array}$ & $\begin{array}{c}\text { Eastern Campus of } \\
\text { Renmin Hospital, Wuhan }\end{array}$ & 27 & 403 & 193 & 210 & $54.75 \pm 4.90$ & 303 & 100 & 20 \\
\hline $\begin{array}{l}\text { Ruan } \\
\text { et al. [21] }\end{array}$ & $\begin{array}{c}\mathrm{RC} \\
\text { (China) }\end{array}$ & $\begin{array}{l}\text { Medical Records of NHC } \\
\text { and China CDC }\end{array}$ & NA & 150 & 102 & 48 & $56.82 \pm 10.95$ & 82 & 68 & 13 \\
\hline $\begin{array}{l}\text { Shi et al. } \\
\text { [22] }\end{array}$ & $\begin{array}{c}\mathrm{RC} \\
\text { (China) }\end{array}$ & Renmin Hospital, Wuhan & 21 & 416 & 205 & 211 & $61.00 \pm 12.47$ & 359 & 57 & 20 \\
\hline $\begin{array}{l}\text { Zhang } \\
\text { et al. [23] }\end{array}$ & $\begin{array}{c}\mathrm{RC} \\
\text { (China) }\end{array}$ & Wuhan No. 1 Hospital & 52 & 48 & 33 & 15 & $64.03 \pm 16.54$ & 31 & 17 & 17 \\
\hline $\begin{array}{l}\text { Zhou } \\
\text { et al. [24] }\end{array}$ & $\begin{array}{c}\text { RC } \\
\text { (China) }\end{array}$ & $\begin{array}{c}\text { Jinyintan Hospital and } \\
\text { Wuhan Pulmonary } \\
\text { Hospital }\end{array}$ & 33 & 191 & 119 & 72 & $56.25 \pm 3.86$ & 137 & 54 & 20 \\
\hline
\end{tabular}

$\mathrm{RC}$, retrospective cohort.

cTn levels compared to surviving patients $(\mathrm{SMD}=2.15$, 95\% CI $0.83-3.47, \quad P=0.001, \quad I^{2}=95 \%, \quad P_{h}<0.0001$, Figure 2(a)). On subgroup analysis, the difference remained significant only for hs-cTnI (SMD $=3.00,95 \%$ CI $2.33-3.66, P<0.0001, I^{2}=85 \%, P$ for heterogeneity $\left.\left(P_{h}\right)=0.0002\right)$. There was no difference in the mean values of NT-proBNP between deceased and surviving patients (Figure 2(b)).

As for secondary cardiac and inflammatory biomarkers, compared to survivors, patients who did not survive had significantly higher levels of IL-6 $(\mathrm{SMD}=3.13,95 \% \mathrm{CI}$ 0.24-6.03, $\left.\quad P=0.03, \quad I^{2}=99 \%, \quad P_{h}<0.0001\right), \quad$ hs-CRP $\left(\mathrm{SMD}=2.78, \quad 95 \% \quad \mathrm{CI} \quad 0.90-4.65, \quad P=0.004, \quad I^{2}=99 \%\right.$, $\left.P_{h}<0.0001\right)$, and CK-MB (SMD $=0.97,95 \%$ CI 0.62-1.32, $P_{h}<0.0001, I^{2}=0 \%, P_{h}=0.46$, Table 2).

3.4. Baseline Parameters and Outcomes in Patients with Elevated or Normal cTn. Data regarding the cutoff values above which cTn was considered elevated were available in seven studies (1380 patients) [18-20, 22-25], whereas detailed clinical characteristics and outcomes in patients with and without cardiac injury were reported in two studies (603 patients) $[18,22]$. The pooled prevalence of elevated cTn on admission was $20.62 \%$ (95\% CI $17.22-24.03, I^{2}=56 \%$, Supplementary Figure S2). At baseline, patients with an initial cardiac injury had higher odds of preexisting CHD $(\mathrm{OR}=8.49, \quad P<0.0001)$, COPD $(\mathrm{OR}=6.50, \quad P=0.0003)$, hypertension $(\mathrm{OR}=5.43, \quad P<0.0001)$, and diabetes $(\mathrm{OR}=2.98, P<0.0001$, Table 3$)$. In addition, patients with elevated cTn had coelevated NT-proBNP (SMD $=5.59,95 \%$ CI $3.00-8.17, P<0.0001)$, CK-MB $(\mathrm{SMD}=6.32,95 \% \mathrm{CI}$ 5.91-6.73, $P<0.0001)$, and hs-CRP $(\mathrm{SMD}=4.69,95 \% \mathrm{CI}$
3.82-5.55, $P<0.0001)$ compared to those with normal cTn values (Table 3 ).

Regarding the outcomes, based on the reported data in seven articles [18-20, 22-25], the risk of death in patients with elevated cTn was significantly higher than that in their peers with normal biomarker values $(\mathrm{RR}=5.28,95 \% \mathrm{CI}$ 3.71-7.51, $P<0.0001, I^{2}=68 \%, P_{h}<0.005$, Figure 3); the risk was also higher when hs-cTnI or cTnT was measured ( $\mathrm{RR}=5.09$ and 6.71, respectively, $P<0.0001$ for both). The studies were distributed symmetrically around the pooled effect estimate, indicating no publication bias (Supplementary Figure S3).

3.5. Dynamic Changes during Hospitalization. The laboratory markers of COVID-19 patients were tracked during hospitalization in two studies [18, 24]. Zhou et al. [24] found that serum hs-cTnI levels increased in nonsurvivors (the median concentrations increased from 57.6 to $290.6 \mathrm{pg} / \mathrm{mL}$ during the period between day 16 and day 22 after the onset of infection, respectively), while they changed only slightly during hospitalization in recovered patients. However, trend changes were not analyzed statistically. In another retrospective analysis, Guo and coworkers [18] revealed statistically significant changes in the median values of plasma cTnT and NT-proBNP during hospitalization and before death compared to values reported on admission. Such temporal changes were not significant in cured patients.

\section{Discussion}

The cardiovascular implications of COVID-19 represent an important aspect of disease pathogenesis and prognosis. The 


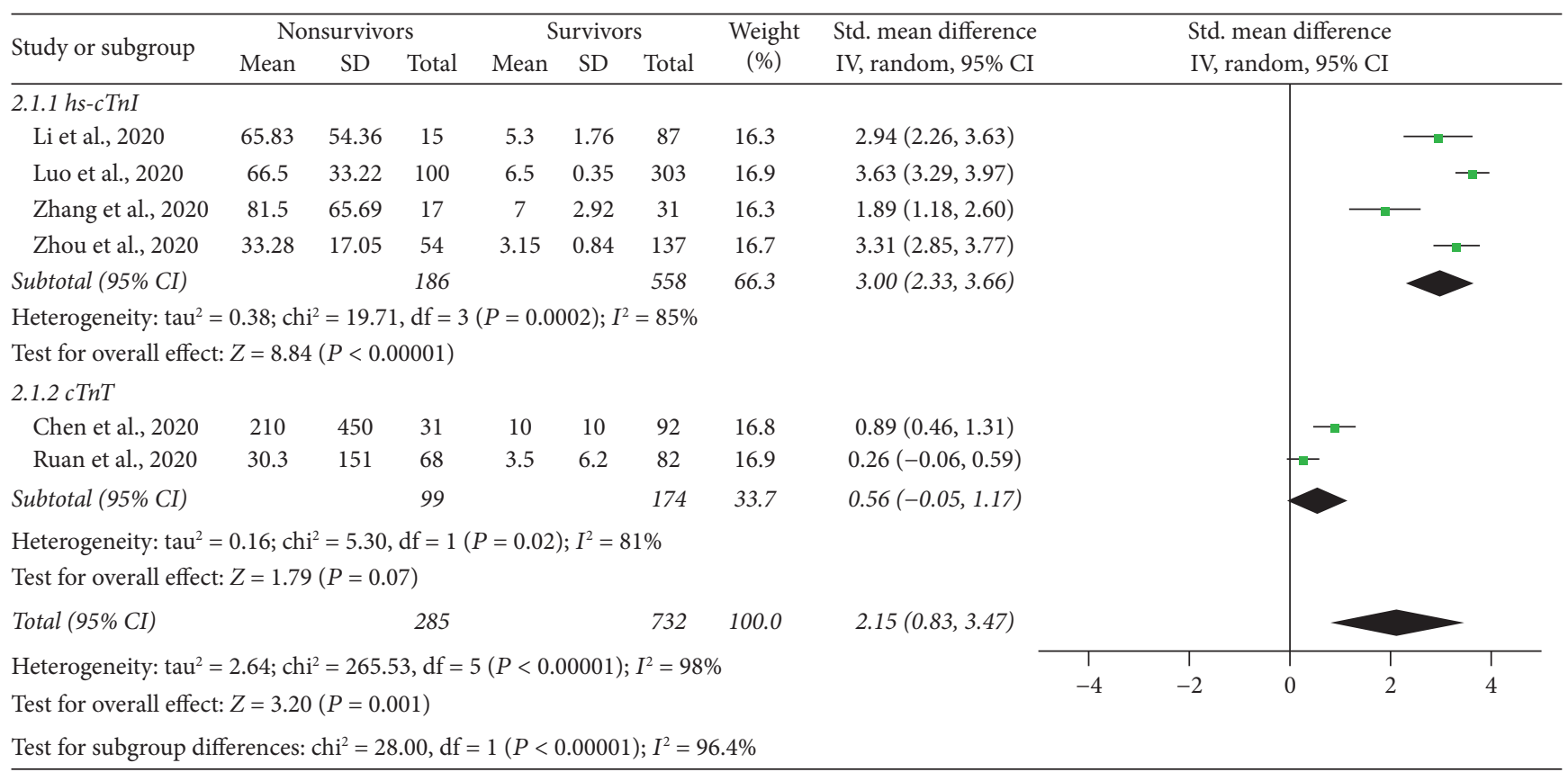

(a)

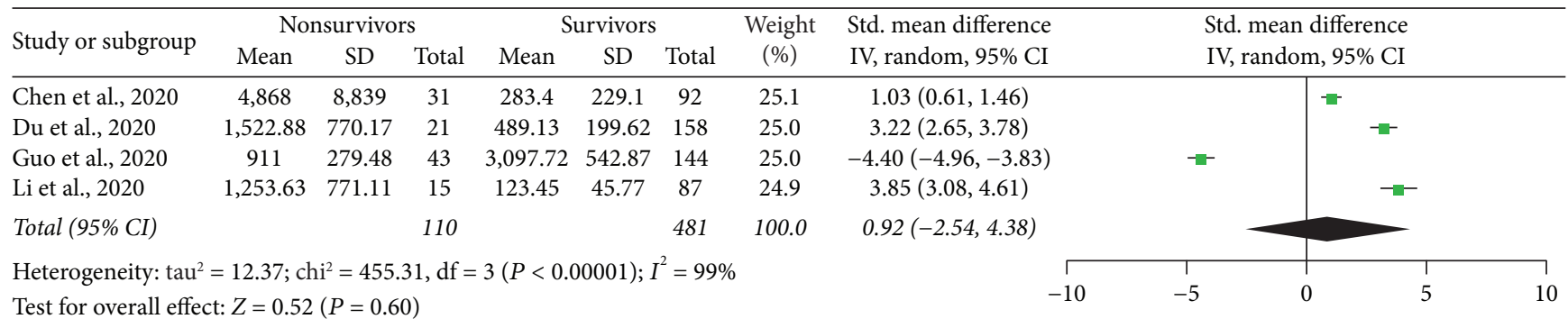

(b)

FIGURE 2: Forest plot showing the difference in the baseline mean values of cTn (a) and NT-proBNP (b) among surviving and nonsurviving COVID-19 patients.

TABLE 2: Baseline differences in secondary cardiac injury biomarkers between COVID-19 survivors and nonsurvivors.

\begin{tabular}{lcccccccc}
\hline \multirow{2}{*}{ Biomarker } & \multirow{2}{*}{ Studies } & \multicolumn{3}{c}{ Patients } & \multicolumn{3}{c}{ Heterogeneity } & \multirow{2}{*}{ SMD (95\% CI) } \\
& & Nonsurvivors & Survivors & Total & Model & $I^{2}(\%)$ & \\
\hline CK-MB & 2 & 48 & 123 & 171 & $\mathrm{~F}$ & 0 & $0.97(0.62,1.32)$ & $<0.0001$ \\
hs-CRP & 5 & 231 & 595 & 826 & $\mathrm{R}$ & 99 & $2.78(0.90,4.65)$ \\
IL-6 & 3 & 137 & 306 & 443 & $\mathrm{R}$ & 99 & $3.13(0.24,6.03)$ & 0.03 \\
Myoglobin & 2 & 89 & 240 & 329 & $\mathrm{R}$ & 99 & $3.00(-1.36,7.36)$ & 0.18 \\
\hline
\end{tabular}

CI, confidence interval; CK-MB, creatine kinase-myocardial bound; CRP, C-reactive protein; F, fixed-effects model; IL-6, interleukin-6; R, random-effects model; SMD, standardized mean difference.

present meta-analysis showed that about one in every five hospitalized patients had cTn levels above the 99th percentile upper reference limit, and elevated cTn on admission was associated with a fivefold increased risk of subsequent death compared to admitted patients with normal cTn $(R R=5.28)$. Moreover, absolute cTn levels became significantly elevated in patients who ultimately died due to COVID-19, and this was also associated with a baseline increase in CK-MB, IL-6, and hs-CRP. In addition to such baseline factors, the development of cardiac injury during hospitalization was evident among nonsurvivors.

Based on these findings, the effects of underlying cardiovascular comorbidities and myocardial damage on the prognosis for COVID-19 patients are significant. To exclude the impact of other confounding factors, multivariable adjusted models have shown independent associations between cardiac injury and mortality, with hazard ratios ranging between 4.26 and 10.90 [22, 23]. Furthermore, 
TABLE 3: Baseline differences between COVID-19 patients with elevated and normal cTn in terms of preexisting comorbidities and the mean values of other cardiac injury biomarkers.

\begin{tabular}{|c|c|c|c|c|c|}
\hline Parameter & Category & Model & $I^{2}(\%)$ & Effect estimate (95\% CI) & $P$ \\
\hline \multirow{6}{*}{ Comorbidities } & Hypertension & $\mathrm{F}$ & 0 & $\mathrm{OR}=5.43(3.60,8.19)$ & $<0.0001$ \\
\hline & Diabetes & $\mathrm{F}$ & 35 & $\mathrm{OR}=2.98(1.84,4.83)$ & $<0.0001$ \\
\hline & Coronary heart disease & $\mathrm{F}$ & 44 & $\mathrm{OR}=8.49(4.85,14.86)$ & $<0.0001$ \\
\hline & COPD & $\mathrm{F}$ & 23 & $\mathrm{OR}=6.50(2.33,18.09)$ & 0.0003 \\
\hline & Malignancy & $\mathrm{R}$ & 72 & $\mathrm{OR}=5.58(0.90,34.70)$ & 0.07 \\
\hline & Chronic kidney disease & $\mathrm{R}$ & 54 & $\mathrm{OR}=4.54(0.80,25.62)$ & 0.09 \\
\hline \multirow{3}{*}{ Biomarkers } & NT-proBNP (pg/mL) & $\mathrm{R}$ & 98 & $\mathrm{SMD}=5.59(3.00,8.17)$ & $<0.0001$ \\
\hline & hs-CRP (mg/L) & $\mathrm{R}$ & 84 & $\mathrm{SMD}=4.69(3.82,5.55)$ & $<0.0001$ \\
\hline & CK-MB (ng/mL) & $\mathrm{F}$ & 0 & $\mathrm{SMD}=6.32(5.91,6.73)$ & $<0.0001$ \\
\hline
\end{tabular}

${ }^{*}$ Results are based on the analysis of 134 and 469 patients with elevated and normal cTn, respectively. CI, confidence interval; CK-MB, creatine kinasemyocardial bound; COPD, chronic obstructive pulmonary disease; F, fixed-effects model; hs-CRP, high-sensitivity C-reactive protein; OR, odds ratio; R, random-effects model; SMD, standardized mean difference.

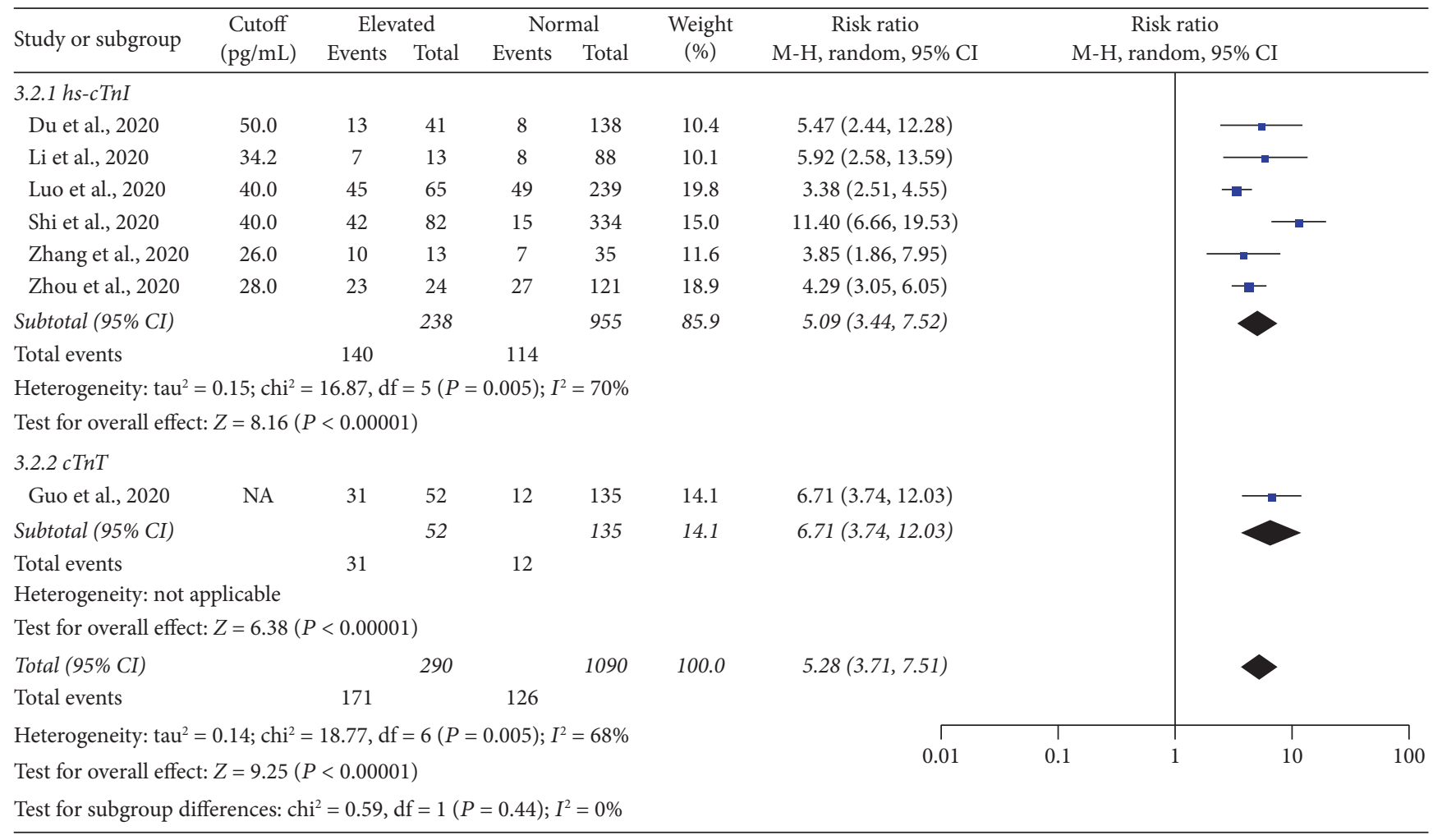

FIgURE 3: A forest plot showing the risk of death among COVID-19 patients with elevated and normal cTn on admission.

recent evidence showed that prior cardioprotective statin therapy has no significant effects on the subsequent COVID19-related mortality [26]. Undoubtedly, cTn is a useful marker for cardiac injury in COVID-19 patients. The role of cTn measurement seems to be more critical in patients with decreased cardiovascular reserve, such as the elderly and those with preexisting cardiovascular comorbidities. Therefore, cTn monitoring, especially hs-cTn, is warranted in selected patients. Patients receiving antiviral drugs could also be monitored, since these medications can lead to arrhythmia, cardiac insufficiency, or cardiac toxicity [27, 28]. It appears that ordering cTn tests in COVID-19 patients should be based on a meticulous estimation of the pretest probability. Notwithstanding the increased sensitivity of hscTn assays, these tests come with a challenging reduction in the diagnostic specificity [29]. As such, the results should be interpreted in the context of preexisting conditions and the findings of electrocardiography and cardiac imaging. This is because false-positive cTn results caused by a low pretest probability would be associated with increased cardiology consults and extensive resource utilization [30].

Nonetheless, cardiac complications have been well established in outbreaks caused by coronaviruses. For example, it has been shown that severe acute respiratory syndrome (SARS) infections could be complicated by acute myocardial infarction, tachyarrhythmias, and signs of heart failure, although no preexisting cardiac diseases had been initially reported $[31,32]$. Arrhythmias were also prevalent among $44 \%$ of patients in early reports following the emerged COVID-19 outbreak in Wuhan, China [33]. Atrial 
arrhythmias are the most commonly reported types, with atrial fibrillation consultations commonly requested during the peak of the COVID-19 outbreak in New York [34]. Other less common arrhythmias included ventricular arrhythmias, ventricular tachycardia storm, atrioventricular block, inappropriate sinus tachycardia, and postural orthostatic tachycardia syndrome [35-37].

Based on the aforementioned observations, it seems that there is a link between myocardial involvement and respiratory infections, and COVID-19 is no exception [38]. Severe hypoxemia resulting from acute respiratory failure contributes to reducing oxygen supply, which would stimulate the sympathetic system and increase myocardial oxygen demand $[39,40]$. Indeed, postmortem studies have revealed that approximately $5-25 \%$ of patients who died from acute respiratory failure had experienced unobserved myocardial infarction [41]. Another possible mechanism is the proinflammatory state, where respiratory infectious agents can elicit an inflammatory pattern in atheromatous plaques [42]. Such a state of inflammation may mediate type I myocardial infarction via upregulating metalloproteinases and peptidases, destabilization of plaques, and thrombus formation [43]. Furthermore, inflammation may induce a prothrombotic state; thus, it promotes coronary thrombosis at sites of plaque detachment [44]. In COVID-19 patients, the pathophysiological changes associated with myocardial ischemia were apparent in the study of Shi et al. [22], where abnormal electrocardiogram (ECG) findings, such as STsegment depression, T-wave depression and inversion, and $\mathrm{Q}$ waves, were reported in all the patients who had exhibited concomitantly elevated cardiac biomarkers.

Importantly, cardiac involvement in patients with COVID-19 may be related to micro- and macrothrombotic complications. A state of hypercoagulation has been reported elsewhere in the literature among patients with influenza $[45,46]$ and COVID-19 [47, 48]. Coagulopathy among COVID-19 patients is typically characterized by an elevated D-dimer concentration, prolonged prothrombin time, and a modest reduction in platelet count [49]. Multiple thrombogenic mechanisms have been proposed, such as stimulation of the complement cascade, RAS dysregulation, and thrombosis triggered by the cytokine storm [50]. Ackermann et al. [48] have shown a significantly higher prevalence of alveolar capillary microthrombi and pulmonary intussusceptive angiogenesis in autopsy specimens of COVID-19 patients compared to those with influenza. There is also growing evidence of the beneficial effects of inhospital heparin on reducing COVID-related in-hospital mortality, whereas prehospitalization oral anticoagulation had had no effects on such a parameter [51]. This would further indicate the important role of microthrombosis in disease severity.

From another point of view, the mechanism of COVID19-related myocardial injury could be explained by myocarditis, and this might be supported by the lack of acute respiratory distress in selected patients. Indeed, two main mechanisms may support such a hypothesis. First, direct viral spread from the respiratory tract through the circulatory or lymphatic systems may be responsible for these changes. The binding of SARS-CoV-2 to ACE-2 receptors, which are highly expressed in the heart [52], provides a rationale for such a hypothesis. Second, an imbalanced response of $\mathrm{T}$ cell populations can induce a rapid and massive production of cytokines, namely, a cytokine storm, causing an immunogenic myocardial injury. Subsequently, prominent cardiovascular changes may occur, such as increased vascular wall permeability, reduced coronary blood flow, coronary plaque destabilization, microthrombogenesis, and myocardial edema $[6,18]$. Unfortunately, when focusing on studies providing detailed analyses of patients with elevated versus normal levels of cardiac injury biomarkers in the present meta-analysis [18, 22], cytokine-level measurement was unavailable, possibly due to logistical limitations.

Nevertheless, the concept of immune-mediated myocarditis could be supported by the fact that IL- 6 was significantly associated with the pre- and postmanagement changes of cTnI levels in a recent case report of COVID-19 complicated with fulminant myocarditis [53]. Finally, as revealed in the current review, the coassociation of hs-CRP as a marker of inflammation with elevated cTn and the significant elevation of IL-6 in nonsurvivors may partly explain the immunogenic responses. The application of the monoclonal anti-IL-6 antibody tocilizumab in the management of COVID-19 pneumonia seems to be a promising solution $[54,55]$, and it is currently being considered in multiple clinical trials on patients who are more likely to develop multiple organ dysfunction and excessive cytokine release (ClinicalTrials.gov identifiers: NCT04332094, NCT04306705, NCT04345445, and NCT04339712).

There are some limitations that should be considered in the present meta-analysis. Patients' data were retrospectively collected, and thus, causal relationships between the risk of mortality and baseline parameters should be interpreted cautiously. Additionally, the included studies were all carried out in China, which might limit the generalizability of the results to other populations worldwide. From another perspective, the rationale of cTn testing was not reported in all studies, and the results of electrocardiography and echocardiography to confirm cardiac injury were not recorded. Data related to the clinical course of COVID-19 patients during the in-hospital stay were only available in two studies $[18,24]$, and thus, the quantitative synthesis of their outcomes was not possible. Importantly, the cause of death may be multifactorial, and it was difficult to emphasize the significance of myocardial injury as the sole and direct cause of death in each individual case. Finally, the small number of studies did not allow further investigation of the causes of heterogeneity via a quantitative analysis (Eggers' test) and the covariates that might have caused changes in the effect measures (meta-regression). These limitations would be resolved via conducting large-sized prospective studies that track the patterns of cTn requesting, monitor cardiac injury during hospitalization with $\mathrm{cTn}$ and radiographic techniques, assess the profiles of cytokines, and determine the causes of death, considering postmortem pathological changes. 


\section{Conclusion}

Approximately one in five COVID-19 patients had a myocardial injury based on cTn testing on admission. Patients presenting with a cardiac injury had a fivefold increase in the risk of death compared to those with normal biomarkers. Deceased patients with a baseline cardiac injury had experienced an abnormal elevation of the inflammatory biomarkers, such as hs-CRP and IL-6, suggesting immune-mediated myocardial damage. Dead patients had also exhibited marked dynamic changes in cTn values during hospitalization, whereas survivors did not. Considering the implementation of high pretest probability, cardiac markers testing, particularly hs-cTnI, is advised on admission for selected patients, such as older adults and those with preexisting cardiovascular comorbidities. In addition, continuous monitoring during hospitalization is important to reduce the risk of cardiovascular complications and death.

\section{Abbreviations}

ACE-2: Angiotensin-converting enzyme 2

CHD: Coronary heart disease

CI: Confidence interval

CK-MB: Creatine kinase-myocardial bound

COPD: Chronic obstructive pulmonary disease

CRP: $\quad$-reactive protein

IL-6: Interleukin-6

MERS: $\quad$ Middle East respiratory syndrome

PRISMA: Preferred Reporting Items for Systematic Reviews and Meta-Analyses

RR: $\quad$ Risk ratio

RT-PCR: Reverse transcriptase polymerase chain reaction

SARS- Severe acute respiratory syndrome coronavirus-

CoV-2: 2

SD: $\quad$ Standard deviation

SMD: $\quad$ Standardized mean difference

STROBE: Strengthening the Reporting of Observational Studies in Epidemiology

WHO: World Health Organization.

\section{Appendix}

\section{The Search Strategy Used in PubMed}

\#1 "troponin" OR "high-sensitivity troponin" OR “ThI” OR “cTnI” OR "hs-cTnI" OR "hypersensitive cTn" OR “TnT" OR "cTnT" OR "hs-cTnT” OR "hypersensitive cTn"

\#2 “NT/proBNP” OR “NT-proBNP” OR “proBNP” OR "natriuretic peptide" OR "N-terminal pro-brain natriuretic peptide"

\#3 “creatinine kinase-myocardial band" OR "Creatine kinase MB" OR "creatinine kinase myocardial band" OR “CK-MB” OR “CKMB”

\#4 \#1 OR \#2 OR \#3

\#5 “Survivor" OR "Survived” OR “Discharged”

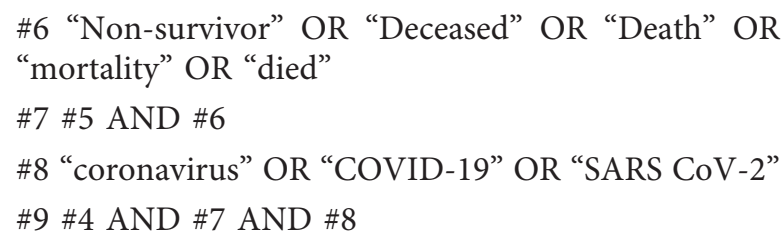

\section{Data Availability}

The data used to support the findings of this study are available from the Department of Family Medicine, King Abdulaziz University, Jeddah, Saudi Arabia.

\section{Ethical Approval}

The protocol of the study was approved by the Research Ethics Committee (REC) of King Abdulaziz University, Jeddah, Saudi Arabia (reference no. 20254).

\section{Conflicts of Interest}

The authors declare that they have no conflicts of interest.

\section{Acknowledgments}

The authors are thankful to the administration of King Abdulaziz University for their support to the team during the work of this article. This research was funded by the Deanship of Scientific Research (DSR), King Abdulaziz University, Jeddah, Saudi Arabia.

\section{Supplementary Materials}

Figure S1: the pooled case fatality rate among COVID-19 patients in the included studies. Figure S2: the pooled prevalence of elevated cTn among hospitalized patients with COVID-19. Figure S3: funnel plots investigating the publication bias for the outcomes of standardized mean differences in cTn (a) and NT-proBNP (b) and the risk of death among patients with normal or abnormal cTn (c). (Supplementary Materials)

\section{References}

[1] P. Okada, R. Buathong, S. Phuygun et al., "Early transmission patterns of coronavirus disease 2019 (COVID-19) in travellers from Wuhan to Thailand, January 2020," Eurosurveillance, vol. 25, no. 8, 2020.

[2] J. F.-W. Chan, K.-H. Kok, Z. Zhu et al., "Genomic characterization of the 2019 novel human-pathogenic coronavirus isolated from a patient with atypical pneumonia after visiting Wuhan," Emerging Microbes \& Infections, vol. 9, no. 1, pp. 221-236, 2020.

[3] P. Zhou, X.-L. Yang, X.-G. Wang et al., "A pneumonia outbreak associated with a new coronavirus of probable bat origin,” Nature, vol. 579, no. 7798, pp. 270-273, 2020.

[4] D. Wrapp, N. Wang, K. S. Corbett et al., "Cryo-EM structure of the 2019-nCoV spike in the prefusion conformation," Science, vol. 367, no. 6483, pp. 1260-1263, 2020.

[5] J. F.-W. Chan, S. Yuan, K.-H. Kok et al., "A familial cluster of pneumonia associated with the 2019 novel coronavirus 
indicating person-to-person transmission: a study of a family cluster," The Lancet, vol. 395, no. 10223, pp. 514-523, 2020.

[6] C. Huang, Y. Wang, X. Li et al., "Clinical features of patients infected with 2019 novel coronavirus in Wuhan, China," The Lancet, vol. 395, no. 10223, pp. 497-506, 2020.

[7] M. Madjid, S. Solomon, and O. Vardeny, ACC Clinical Bulletin Focuses on Cardiac Implications of Coronavirus (COVID-19), American College of Cardiology Science and Quality Committee, Washington, DC, USA, 2020, https://www.acc.org/latest-incardiology/articles/2020/02/13/12/42/acc-clinical-bulletin-focuseson-cardiac-implications-of-coronavirus-2019-ncov.

[8] D. Hof and A. von Eckardstein, "High-sensitivity troponin assays in clinical diagnostics of acute coronary syndrome," Methods in Molecular Biology (Clifton, NJ), vol. 1929, pp. 645-662, 2019.

[9] Z. Cao, Y. Jia, and B. Zhu, "BNP and NT-proBNP as diagnostic biomarkers for cardiac dysfunction in both clinical and forensic medicine," International Journal of Molecular Sciences, vol. 20, no. 8, p. 1820, 2019.

[10] D. Moher, A. Liberati, J. Tetzlaff, D. G. Altman, and P. Group, "Preferred reporting items for systematic reviews and metaanalyses: the PRISMA statement," PLoS Medicine, vol. 6, no. 7, Article ID e1000097, 2009.

[11] World Health Organization, Clinical Management of Severe Acute Respiratory Infection when COVID-19 Is Suspected: Interim Guidance Geneva, WHO, Geneva, Switzerland, 2020, https://www.who.int/publications-detail/clinical-managementof-severe-acute-respiratory-infection-when-novel-coronavirus(ncov)-infection-is-suspected.

[12] X.-Y. Wang, F. Zhang, C. Zhang, L.-R. Zheng, and J. Yang, "The biomarkers for acute myocardial infarction and heart failure," BioMed Research International, vol. 2020, Article ID 2018035, 14 pages, 2020.

[13] X. Wan, W. Wang, J. Liu, and T. Tong, "Estimating the sample mean and standard deviation from the sample size, median, range and/or interquartile range," BMC Medical Research Methodology, vol. 14, no. 1, p. 135, 2014.

[14] E. Von Elm, D. G. Altman, M. Egger, S. J. Pocock, P. C. Gøtzsche, and J. P. Vandenbroucke, "The strengthening the reporting of observational studies in epidemiology (STROBE) statement: guidelines for reporting observational studies," Annals of Internal Medicine, vol. 147, no. 8, pp. 573-577, 2007.

[15] H. Hui, Y. Zhang, X. Yang et al., "Clinical and radiographic features of cardiac injury in patients with 2019 novel coronavirus pneumonia," 2020, https://www.medrxiv.org/ content/10.1101/2020.02.24.20027052v1.full.pdf.

[16] C. Wu, X. Hu, J. Song et al., "Heart injury signs are associated with higher and earlier mortality in coronavirus disease 2019 (COVID-19)," 2020, https://www.medrxiv.org/content/10. $1101 / 2020.02 .26 .20028589 \mathrm{v} 1$.

[17] M. Chen, Y. Fan, X. Wu et al., "Clinical characteristics and risk factors for fatal outcome in patients with 2019-coronavirus infected disease (COVID-19) in Wuhan, China," SSRN Electronic Journal, 2020.

[18] T. Guo, Y. Fan, M. Chen et al., "Cardiovascular implications of fatal outcomes of patients with coronavirus disease 2019 (COVID-19)," JAMA Cardiology, vol. 5, no. 7, pp. 811-818, 2020.

[19] K. Li, D. Chen, S. Chen et al., "Radiographic findings and other predictors in adults with Covid-19," 2020, https://www. medrxiv.org/content/10.1101/2020.03.23.20041673v1.

[20] X. Luo, H. Xia, W. Yang et al., "Characteristics of patients with COVID-19 during epidemic ongoing outbreak in Wuhan,
China," 2020, https://www.medrxiv.org/content/10.1101/ 2020.03.19.20033175v1.

[21] Q. Ruan, K. Yang, W. Wang, L. Jiang, and J. Song, "Clinical predictors of mortality due to COVID-19 based on an analysis of data of 150 patients from Wuhan, China," Intensive Care Medicine, vol. 46, no. 5, pp. 846-848, 2020.

[22] S. Shi, M. Qin, B. Shen et al., "Association of cardiac injury with mortality in hospitalized patients with COVID-19 in Wuhan, China," JAMA Cardiology, vol. 5, no. 7, pp. 802-810, 2020.

[23] F. Zhang, D. Yang, J. Li et al., "Myocardial injury is associated with in-hospital mortality of confirmed or suspected COVID19 in Wuhan, China: a single center retrospective cohort study," 2020, https://www.medrxiv.org/content/10.1101/2020. 03.21.20040121v1.

[24] F. Zhou, T. Yu, R. Du et al., "Clinical course and risk factors for mortality of adult inpatients with COVID-19 in Wuhan, China: a retrospective cohort study," The Lancet, vol. 395, no. 10229, pp. 1054-1062, 2020.

[25] R.-H. Du, L.-R. Liang, C.-Q. Yang et al., "Predictors of mortality for patients with COVID-19 pneumonia caused by SARS-CoV-2: a prospective cohort study," European Respiratory Journal, vol. 55, no. 5, Article ID 2000524, 2020.

[26] G. Mitacchione, M. Schiavone, A. Curnis et al., "Impact of prior statin use on clinical outcomes in COVID-19 patients: data from tertiary referral hospitals during COVID-19 pandemic in Italy," Journal of Clinical Lipidology, vol. 15, no. 1, pp. $68-78,2020$.

[27] C. Ucciferri, A. Occhionero, J. Vecchiet, and K. Falasca, "Cardiac toxicity associated with HCV direct antiviral agents," Mediterranean Journal of Hematology and Infectious Diseases, vol. 10, Article ID e2018069, 2018.

[28] M. Sakabe, R. Yoshioka, and A. Fujiki, "Sick sinus syndrome induced by interferon and ribavirin therapy in a patient with chronic hepatitis C," Journal of Cardiology Cases, vol. 8, no. 6, pp. 173-175, 2013.

[29] S. Bhoi, S. Galwankar, S. Vankar, and P. Verma, "High sensitivity troponins and conventional troponins at the bedside," International Journal of Critical Illness and Injury Science, vol. 4, no. 3, pp. 253-256, 2014.

[30] S. Talebi, R. M. Ferra, S. Tedla et al., "Hazards with ordering troponin in patients with low pretest probability of acute coronary syndrome," The American Journal of Emergency Medicine, vol. 33, no. 9, pp. 1258-1260, 2015.

[31] C.-M. Yu, R. S. M. Wong, E. B. Wu et al., "Cardiovascular complications of severe acute respiratory syndrome," Postgraduate Medical Journal, vol. 82, no. 964, pp. 140-144, 2006.

[32] J. S. M. Peiris, C. M. Chu, V. C. C. Cheng et al., "Clinical progression and viral load in a community outbreak of coronavirus-associated SARS pneumonia: a prospective study," The Lancet, vol. 361, no. 9371, pp. 1767-1772, 2003.

[33] D. Wang, B. Hu, C. Hu et al., "Clinical characteristics of 138 hospitalized patients with 2019 novel coronavirus-infected pneumonia in Wuhan, China," Jama, vol. 323, no. 11, pp. 1061-1069, 2020.

[34] J. P. Berman, M. P. Abrams, A. Kushnir et al., "Cardiac electrophysiology consultative experience at the epicenter of the COVID-19 pandemic in the United States," Indian Pacing and Electrophysiology Journal, vol. 20, no. 6, pp. 250-256, 2020.

[35] A. D. Desai, B. C. Boursiquot, L. Melki, and E. Y. Wan, "Management of arrhythmias associated with COVID-19," Current Cardiology Reports, vol. 23, pp. 1-9, 2021.

[36] G. Mitacchione, M. Schiavone, A. Gasperetti, and G. B. Forleo, "Ventricular tachycardia storm management in a 
COVID-19 patient: a case report," European Heart JournalCase Reports, vol. 4, pp. 1-6, 2020.

[37] K. J. Clerkin, J. A. Fried, J. Raikhelkar et al., "COVID-19 and cardiovascular disease," Circulation, vol. 141, no. 20, pp. 1648-1655, 2020.

[38] M. Schiavone, C. Gobbi, G. Biondi-Zoccai et al., "Acute coronary syndromes and COVID-19: exploring the uncertainties," Journal of Clinical Medicine, vol. 9, no. 6, p. 1683, 2020.

[39] Y. Sandoval and A. S. Jaffe, "Type 2 myocardial infarction," Journal of the American College of Cardiology, vol. 73, no. 14, pp. 1846-1860, 2019.

[40] J. A. Davidson and C. Warren-Gash, "Cardiovascular complications of acute respiratory infections: current research and future directions," Expert Review of Anti-infective Therapy, vol. 17, no. 12, pp. 939-942, 2019.

[41] A. Soeiro, A. Ruppert, M. Canzian, V. Capelozzi, and C. Serrano-Junior Jr., "Postmortem diagnosis of acute myocardial infarction in patients with acute respiratory failure-demographics, etiologic and pulmonary histologic analysis," Clinics, vol. 67, no. 3, pp. 213-217, 2012.

[42] A. Mauriello, G. Sangiorgi, S. Fratoni et al., "Diffuse and active inflammation occurs in both vulnerable and stable plaques of the entire coronary tree," Journal of the American College of Cardiology, vol. 45, no. 10, pp. 1585-1593, 2005.

[43] P. Libby, "Mechanisms of acute coronary syndromes and their implications for therapy," New England Journal of Medicine, vol. 368, no. 21, pp. 2004-2013, 2013.

[44] R. E. Harskamp and M. W. Van Ginkel, “Acute respiratory tract infections: a potential trigger for the acute coronary syndrome," Annals of Medicine, vol. 40, no. 2, pp. 121-128, 2008.

[45] J. C. Kwong, K. L. Schwartz, M. A. Campitelli et al., “Acute myocardial infarction after laboratory-confirmed influenza infection," New England Journal of Medicine, vol. 378, no. 4, pp. 345-353, 2018.

[46] M. Sugiura, K. Hiraoka, S.-i. Ohkawa, K. Ueda, T. Matsuda, and M. Murakami, "A clinicopathological study on cardiac lesions in 64 cases of disseminated intravascular coagulation," Japanese Heart Journal, vol. 18, no. 1, pp. 57-69, 1977.

[47] S. Cui, S. Chen, X. Li, S. Liu, and F. Wang, "Prevalence of venous thromboembolism in patients with severe novel coronavirus pneumonia," Journal of Thrombosis and Haemostasis, vol. 18, no. 6, pp. 1421-1424, 2020.

[48] M. Ackermann, S. E. Verleden, M. Kuehnel et al., "Pulmonary vascular endothelialitis, thrombosis, and angiogenesis in Covid-19," New England Journal of Medicine, vol. 383, no. 2, pp. 120-128, 2020.

[49] M. Levi, J. Thachil, T. Iba, and J. H. Levy, "Coagulation abnormalities and thrombosis in patients with COVID-19," The Lancet Haematology, vol. 7, no. 6, pp. e438-e440, 2020.

[50] T. C. Hanff, A. M. Mohareb, J. Giri, J. B. Cohen, and J. A. Chirinos, "Thrombosis in COVID-19," American Journal of Hematology, vol. 95, no. 12, pp. 1578-1589, 2020.

[51] M. Schiavone, A. Gasperetti, M. Mancone et al., "Oral anticoagulation and clinical outcomes in COVID-19: an Italian multicenter experience," International Journal of Cardiology, vol. 323, pp. 276-280, 2021.

[52] L. Chen, X. Li, M. Chen, Y. Feng, and C. Xiong, "The ACE2 expression in human heart indicates new potential mechanism of heart injury among patients infected with SARS-CoV2," Cardiovascular Research, vol. 116, no. 6, pp. 1097-1100, 2020.
[53] J.-H. Zeng, Y.-X. Liu, J. Yuan et al., "First case of COVID-19 complicated with fulminant myocarditis: a case report and insights," Infection, vol. 48, no. 5, pp. 773-777, 2020.

[54] P. Luo, Y. Liu, L. Qiu, X. Liu, D. Liu, and J. Li, “Tocilizumab treatment in COVID-19: a single center experience," Journal of Medical Virology, vol. 92, no. 7, pp. 814-818, 2020.

[55] B. Liu, M. Li, Z. Zhou, X. Guan, and Y. Xiang, "Can we use interleukin-6 (IL-6) blockade for coronavirus disease 2019 (COVID-19)-induced cytokine release syndrome (CRS)?" Journal of Autoimmunity, vol. 111, Article ID 102452, 2020. 